\title{
American Diabetes Association Framework for Glycemic Control in Older Adults: Implications for Risk of Hospitalization and Mortality
}

Authors: Mary R. Rooney, Olive Tang, Justin B. Echouffo Tcheugui, Pamela L. Lutsey, Morgan E. Grams, B. Gwen Windham, Elizabeth Selvin

\section{Contents}

Supplemental Table S1. ICD-9 codes used to define American Diabetes Association comorbidities

Supplemental Table S2. Mortality and total hospitalizations in older adults with diabetes according to A1C categories, overall and by comorbid health status: The Atherosclerosis Risk in Communities (ARIC) Study, 2011 to 2018

Supplemental Table S3. Tabulation of health status categories according to glucose-lowering medication use, overall and in individuals with $\mathrm{A} 1 \mathrm{C}$ below $7 \%$

Supplemental Table S4. Mortality and total hospitalizations in older adults with diabetes restricted to insulin or sulfonylurea users, according to comorbid health status, overall and by A1C categories: The ARIC Study, 2011 to 2018

Supplemental Figure S1. Study participant flow chart

Supplemental Figure S2. Kaplan Meier survival curves of mortality in older adults with diabetes according to health status: The ARIC Study, 2011 to 2018

Supplemental Figure S3. Adjusted hazard ratio of the association of individual comorbidities with allcause mortality: The ARIC Study, 2011 to 2018

Supplemental Figure S4. Adjusted incidence rate ratio of the association of individual comorbidities with total count of hospitalizations: The ARIC Study, 2011 to 2018 
Supplemental Table S1. ICD-9 codes used to define American Diabetes Association comorbidities

\begin{tabular}{|l|l|l|}
\hline Comorbidity & ICD-9 Codes Used & CMS Claims Used \\
\hline Arthritis & $714 . X 715 . X$ & Yes \\
\hline Emphysema and COPD & 416.8416 .9490491492493494495496500501502503 & \\
& 504505506.4508 .1508 .8 & \\
\hline History of falls & E880.0 E880.1 E880.9 E881.0 E881.1 E882 E883.0 E883.1 & Yes \\
& E883.2 E883.9 E884.0 E884.1 E884.2 E884.3 E884.4 E884.5 & \\
& E884.6 E884.9 E885 E885.0 E885.1 E885.2 E885.3 E885.4 & \\
& E885.9 E886.0 E886.9 E888 E888.0 E888.1 E888.8 E888.9 & \\
& E968.1 E987.0 E987.1 E987.2 E987.9 & Yes \\
\hline Hypoglycemia & 250.3250 .8251251 .1251 .2270 .3775775 .6962 .3 & Yes \\
\hline Incontinence & 788.3 & \multicolumn{2}{|l|}{} \\
\hline Chronic kidney disease & 585.3585 .4585 .5585 .6585 .9 & . \\
\hline
\end{tabular}

Abbreviations: ICD, International Classification of Diseases; CMS, Centers for Medicare and Medicaid;

COPD, chronic obstructive pulmonary disease 
Supplemental Table S2. Mortality and total hospitalizations in older adults with diabetes according to A1C categories, overall and by comorbid health status: The Atherosclerosis Risk in Communities (ARIC) Study, 2011 to 2018

\begin{tabular}{|c|c|c|c|c|}
\hline \multicolumn{2}{|c|}{ A1C \& health status categories } & $\begin{array}{c}\mathrm{N} \\
\text { total }\end{array}$ & $\begin{array}{c}\text { All-Cause Mortality } \\
\text { HR }(95 \% \mathrm{Cl})^{*}\end{array}$ & $\begin{array}{c}\text { Total Hospitalizations } \\
\text { IRR }(95 \% \mathrm{Cl})^{\star}\end{array}$ \\
\hline \multirow[t]{3}{*}{$\mathrm{A} 1 \mathrm{C}<7 \%$} & Healthy & 433 & 1 (Ref) & 1 (Ref) \\
\hline & Complex/Intermediate & 547 & $2.12(1.46,3.07)$ & $1.92(1.61,2.28)$ \\
\hline & Very Complex/Poor & 337 & $4.60(3.15,6.73)$ & $3.40(2.78,4.15)$ \\
\hline \multirow[t]{3}{*}{ A1C $7-<8 \%$} & Healthy & 101 & 1 (Ref) & 1 (Ref) \\
\hline & Complex/Intermediate & 143 & $1.90(0.99,3.63)$ & $2.79(1.98,3.92)$ \\
\hline & Very Complex/Poor & 90 & $3.48(1.77,6.83)$ & $3.70(2.53,5.42)$ \\
\hline \multirow[t]{3}{*}{$\mathrm{A} 1 \mathrm{C} \geq 8 \%$} & Healthy & 48 & 1 (Ref) & 1 (Ref) \\
\hline & Complex/Intermediate & 76 & $1.21(0.47,3.14)$ & $3.02(1.88,4.85)$ \\
\hline & Very Complex/Poor & 66 & $3.33(1.33,8.34)$ & $3.96(2.39,6.56)$ \\
\hline \multicolumn{5}{|c|}{ Alternative A1C Cut-points } \\
\hline \multirow[t]{3}{*}{$\mathrm{A} 1 \mathrm{C}<6 \%$} & Healthy & 181 & 1 (Ref) & 1 (Ref) \\
\hline & Complex/Intermediate & 219 & $2.76(1.57,4.86)$ & $1.88(1.42,2.49)$ \\
\hline & Very Complex/Poor & 135 & $5.00(2.76,9.08)$ & $3.44(2.48,4.77)$ \\
\hline \multirow[t]{3}{*}{$\mathrm{A} 1 \mathrm{C} \geq 9 \%$} & Healthy & 24 & 1 (Ref) & 1 (Ref) \\
\hline & Complex/Intermediate & 36 & $4.40(0.54,36.10)$ & $4.08(1.98,8.39)$ \\
\hline & Very Complex/Poor & 31 & $8.68(1.10,68.60)$ & $4.61(2.06,10.30)$ \\
\hline
\end{tabular}

Abbreviations: $\mathrm{HR}$, hazard ratio; IRR, incidence rate ratio

${ }^{*}$ Adjusted for age, sex, race-center 
Supplemental Table S3. Tabulation of health status categories according to glucose-lowering medication use, overall and in individuals with A1C below 7\%

\begin{tabular}{|l|c|c|c|c|}
\hline & Overall & $\begin{array}{c}\text { No glucose- } \\
\text { lowering } \\
\text { medication }\end{array}$ & $\begin{array}{c}\text { Non-insulin/ } \\
\text { sulfonylurea } \\
\text { medication only }\end{array}$ & $\begin{array}{c}\text { Insulin or } \\
\text { sulfonylurea }\end{array}$ \\
\hline Health status categories & 582 & $257(44.2)$ & $159(27.3)$ & $166(28.5)$ \\
\hline Healthy & 766 & $289(37.7)$ & $192(25.1)$ & $285(37.2)$ \\
\hline Complex/Intermediate & 493 & $166(33.7)$ & $115(23.3)$ & $212(43.0)$ \\
\hline Very Complex/Poor & & & & \\
\hline & & & & \\
\hline $\begin{array}{l}\text { Health status categories } \\
\text { in people with A1C <7\% }\end{array}$ & 433 & $238(55.0)$ & $118(27.3)$ & $77(17.8)$ \\
\hline Healthy & 547 & $272(49.7)$ & $146(26.7)$ & $129(23.6)$ \\
\hline Complex/Intermediate & 337 & $148(43.9)$ & $90(26.7)$ & $99(29.4)$ \\
\hline Very Complex/Poor & &
\end{tabular}

$\mathrm{N}($ row \%) 
Supplemental Table S4. Mortality and total hospitalizations in older adults with diabetes restricted to insulin or sulfonylurea users, according to comorbid health status, overall and by A1C categories: The ARIC Study, 2011 to 2018

\begin{tabular}{|c|c|c|c|}
\hline \multicolumn{2}{|c|}{ Insulin or Sulfonylurea Users $(\mathrm{N}=663)$} & $\begin{array}{c}\text { Mortality } \\
\text { HR }(95 \% \text { CI)* }\end{array}$ & $\begin{array}{c}\text { Total } \\
\text { Hospitalizations } \\
\text { IRR }(95 \% \mathrm{Cl})^{\star} \\
\end{array}$ \\
\hline \multicolumn{4}{|c|}{ Health status categories ${ }^{*}$} \\
\hline \multicolumn{2}{|l|}{ Healthy } & 1 (Ref) & 1 (Ref) \\
\hline \multicolumn{2}{|l|}{ Complex/Intermediate } & $1.96(1.20,3.20)$ & $2.71(2.10,3.50)$ \\
\hline \multicolumn{2}{|l|}{ Very Complex/Poor } & $3.84(2.34,6.30)$ & $3.80(2.89,5.00)$ \\
\hline \multicolumn{4}{|c|}{ Health status \& A1C categories } \\
\hline \multirow[t]{3}{*}{ Healthy } & $\mathrm{A} 1 \mathrm{C}<7 \%$ & 1 (Ref) & 1 (Ref) \\
\hline & A1C $7-<8 \%$ & $1.13(0.42,3.07)$ & $1.25(0.74,2.12)$ \\
\hline & $\mathrm{A} 1 \mathrm{C} \geq 8 \%$ & $1.72(0.55,5.35)$ & $1.33(0.73,2.43)$ \\
\hline \multirow[t]{3}{*}{ Complex/Intermediate } & $\mathrm{A} 1 \mathrm{C}<7 \%$ & 1 (Ref) & 1 (Ref) \\
\hline & A1C $7-<8 \%$ & $1.04(0.61,1.77)$ & $1.10(0.79,1.53)$ \\
\hline & $\mathrm{A} 1 \mathrm{C} \geq 8 \%$ & $1.18(0.63,2.20)$ & $1.38(0.95,2.01)$ \\
\hline \multirow[t]{3}{*}{ Very Complex/Poor } & $\mathrm{A} 1 \mathrm{C}<7 \%$ & 1 (Ref) & 1 (Ref) \\
\hline & A1C $7-<8 \%$ & $0.98(0.57,1.68)$ & $0.77(0.53,1.11)$ \\
\hline & $\mathrm{A} 1 \mathrm{C} \geq 8 \%$ & $1.59(0.91,2.78)$ & $0.91(0.61,1.35)$ \\
\hline
\end{tabular}

Abbreviations: HR, hazard ratio; IRR, incidence rate ratio

${ }^{*}$ Adjusted for age, sex, race-center 
Supplemental Figure S1. Study participant flow chart

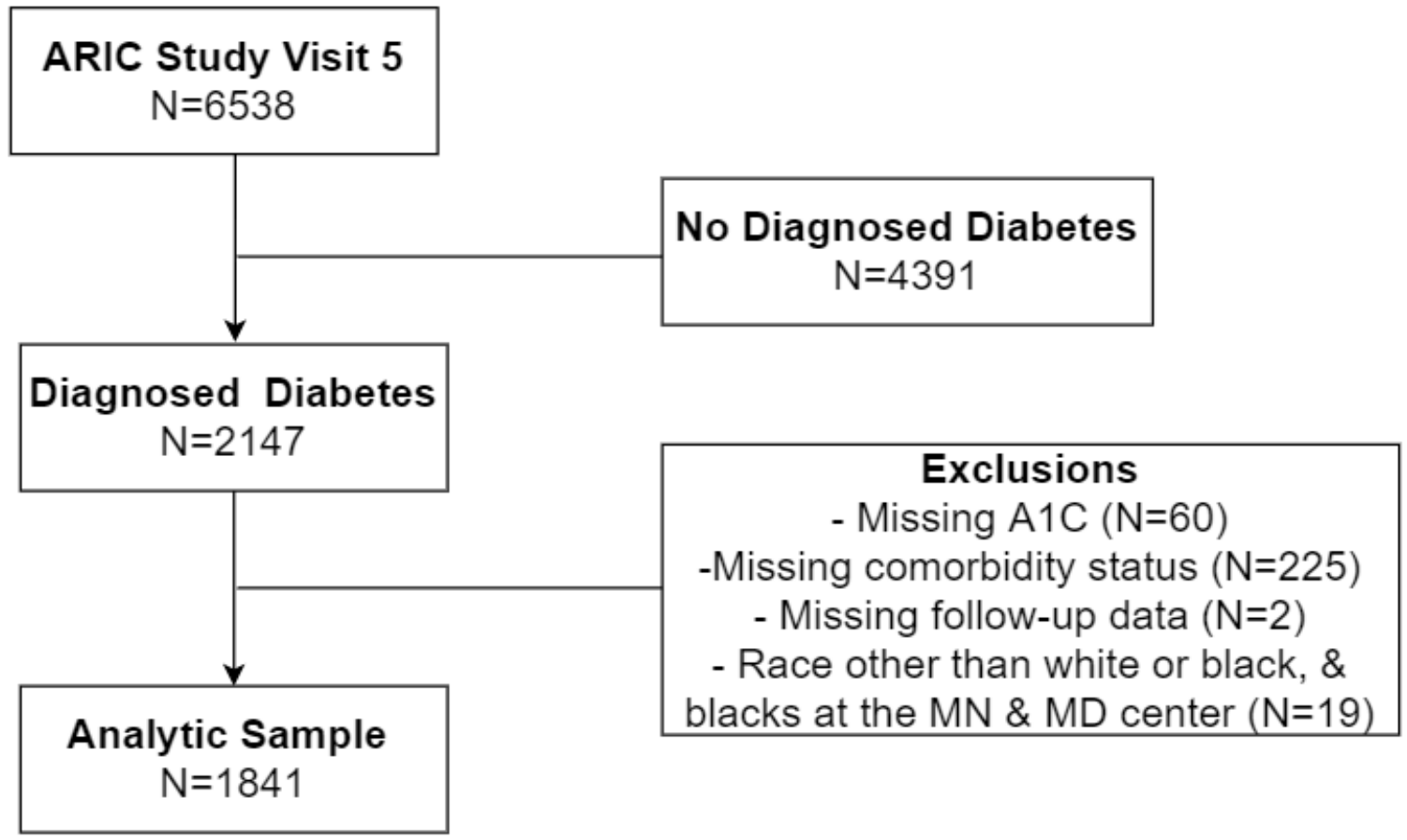


Supplemental Figure S2. Kaplan Meier survival curves of mortality in older adults with diabetes according to health status: The ARIC Study, 2011 to 2018

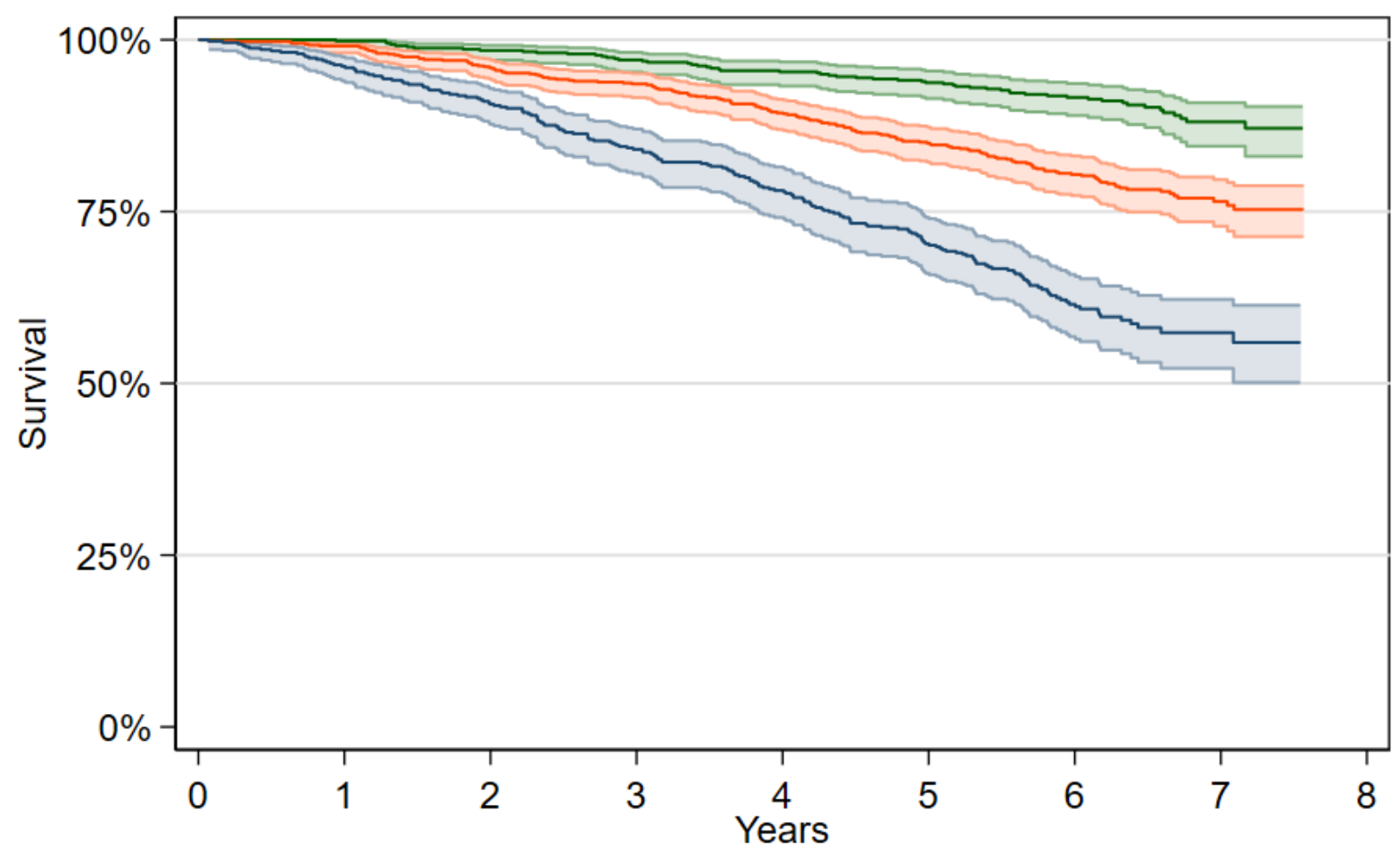


Supplemental Figure S3. Adjusted hazard ratio of the association of individual comorbidities with allcause mortality: The ARIC Study, 2011 to 2018

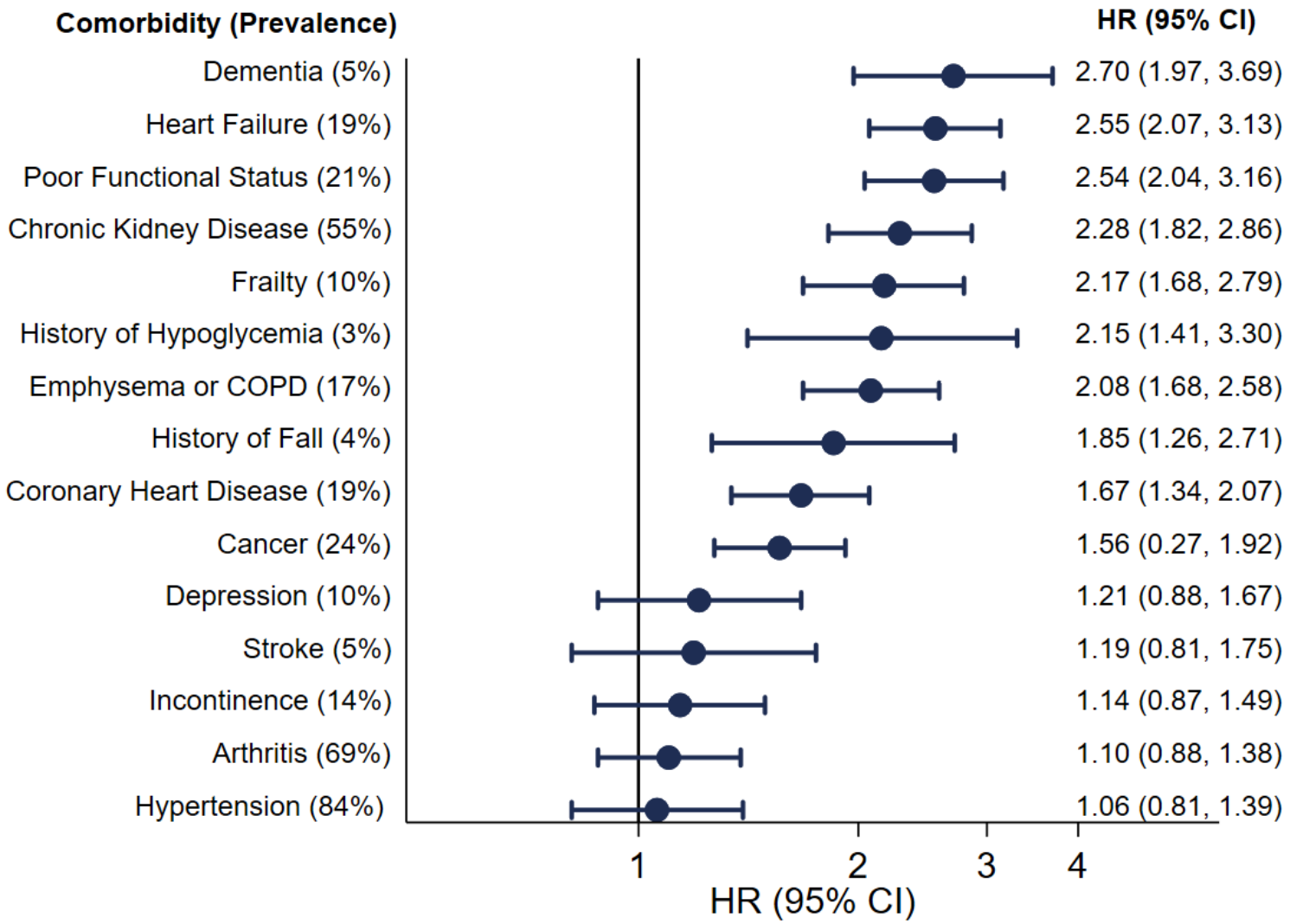

Abbreviations: HR, hazard ratio; COPD, chronic obstructive pulmonary disease. HR adjusted for age, sex, race-center 
Supplemental Figure S4. Adjusted incidence rate ratio of the association of individual comorbidities with total count of hospitalizations: The ARIC Study, 2011 to 2018

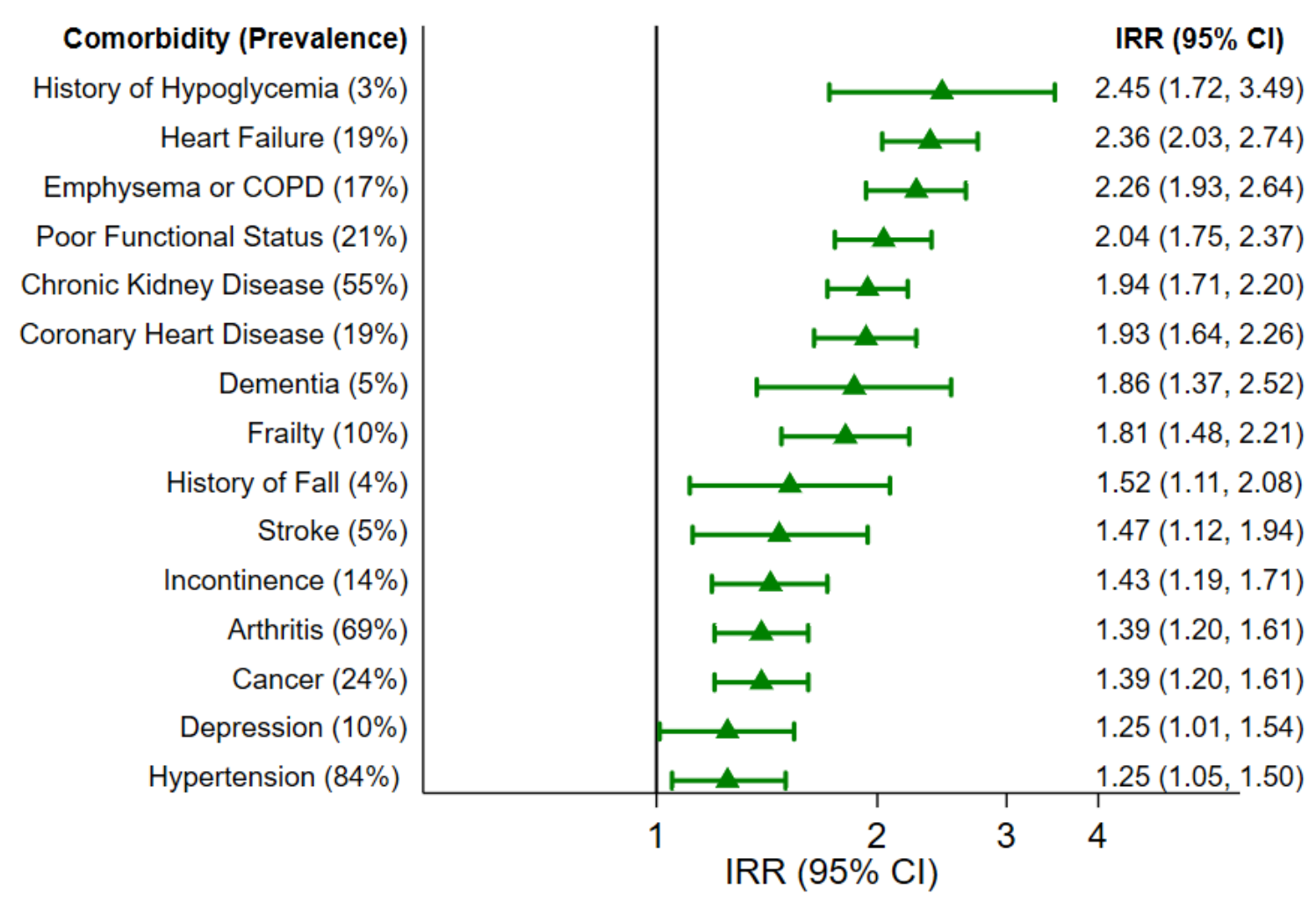

Abbreviations: IRR, incidence rate ratio; COPD, chronic obstructive pulmonary disease. IRR adjusted for age, sex, race-center 